\title{
Thermal model description of the particle spectra in the few-GeV energy regime
}

\author{
Szymon Harabasz ${ }^{1,3, *}$, Wojciech Florkowski ${ }^{2}$, Tetyana Galatyuk $^{1,3}$, Matgorzata Gumberidze $^{3}$, \\ Radostaw Ryblewski ${ }^{4}$, Piotr Salabura ${ }^{5}$, and Joachim Stroth $^{3,6}$ \\ ${ }^{1}$ Institut für Kernphysik, Technische Universitä Darmstadt, 64289 Darmstadt, Germany \\ ${ }^{2}$ Institute of Theoretical Physics, Jagiellonian University, 30-348 Kraków, Poland \\ ${ }^{3}$ GSI Helmholtzzentrum für Schwerionenforschung GmbH, 64291 Darmstadt, Germany \\ ${ }^{4}$ Institute of Nuclear Physics, Polish Academy of Sciences, 31-342 Kraków, Poland \\ ${ }^{5}$ Marian Smoluchowski Institute of Physics, Jagiellonian University, 30-348 Kraków, Poland \\ ${ }^{6}$ Institut für Kernphysik, Goethe-Universität, 60438 Frankfurt, Germany
}

\begin{abstract}
It has been demonstrated that Statistical Hadronization Model fits perfectly to particle yields at freeze-out in heavy-ion and hadron collisions at LHC, RHIC and SPS, where quark-gluon plasma is created. It is however entirely not clear if particles emitted in the few-GeV energy regime can be understood as emerging from thermalized hadronic medium. Our recent work suggests that this might be the case. By implementing appropriate fireball geometry and expansion pattern in the THERMINATOR (THERMal heavy IoN generATOR) it was possible to describe not only yields, but also the spectra of most abundant particles measured at GSI SIS18. Most of the latter are pure prediction of the model. We present details of the model and extended comparison with experimental data and discuss further developments.
\end{abstract}

\section{Introduction}

Models based on statistical hadronization have been very successful in describing over many orders of magnitude the multiplicities of hadrons produced in different collision processes, in particular in heavy-ion collisions (HICs), see e.g. [1] and references therein. This is possible with just a few free thermodynamic parameters (temperature, fugacities, system's volume), whose fitted values allow to relate collision energy and system size to the location in the QCD phase diagram. At the same time, the assumption of local thermodynamic equilibrium makes it possible to use concepts originating from fluid dynamics to describe the evolution of hot and dense fireball of QCD matter produced in HICs.

Collisions at energy of few $\mathrm{GeV}$ probe the phase diagram region of moderate temperature (tens of $\mathrm{MeV}$ ) and high baryochemical potential $\mu_{\mathrm{B}}$ (close to the nucleon mass). However, it is debated if at such collision energies matter does reach local thermal equilibrium. Intuitively, on one hand the number of newly produced particles is small ( $\sim 40$ pions), on the other hand the degree of baryon stopping is high and furthermore the lifetime of the fireball is relatively long. A possible way to test this is to compare the kinematic distributions of particles predicted by the thermal model accompanied with hydro-inspired description of the fireball expansion to experimental results.

\footnotetext{
*e-mail: S.Harabasz@gsi.de
} 


\section{Model and results}

In this work we extend the THERMal heavy IoN generATOR (THERMINATOR) [2] to make it applicable for comparison with protons and pions spectra measured by the HADES Collaboration in $10 \%$ most central $\mathrm{Au}+\mathrm{Au}$ collisions at $\sqrt{s_{\mathrm{NN}}}=2.42 \mathrm{GeV}[4,5]$. In our calculation we assume that the chemical freeze-out (which we define as the moment when final abundances of particles, not just flavor content, are fixed) coincides with the kinetic freeze-out (when the momentum distributions are fixed). This assumption was successful in describing the RHIC measurements [3]. We use the Cooper-Frye formula for particle production [6]. The five parameters of the thermal distribution and the total volume we determine by fitting to the measured abundances of six particles: in addition to $p$ and $\pi^{ \pm}$these are $\Lambda$ [7] and $K^{ \pm}$[8]. We add the protons bound in light nuclear clusters to the ones measured as free, assuming implicitly that the cluster formation proceeds through the coalescence mechanism after the freeze-out. We assume the spherical symmetry of the fireball and its radial expansion following Siemens and Rasmussen [9] but with Hubble-like expansion velocity profile $\beta(r)=\tanh (H r)$. The parameter $H$ is determined with a fit to a $p$ transverse mass spectrum at mid-rapidity. Shapes of all the remaining particle spectra do not enter the fit in any way and can therefore be considered as predictions of the model. Finally, we include the correct mass distribution of the most relevant resonance, $\Delta(1232)$, as a derivative of the empirical phase shift taken from ref. [10]. The details of the model and the complete set of results can be found in [11], all the parameters are also displayed in the upper row of Fig. 3.

\section{Discussion}

With the help of an event generator it is possible to check the influence of different assumptions on final results. In Fig. 1 we show a comparison of experimentally measured $m_{t}$ spectra
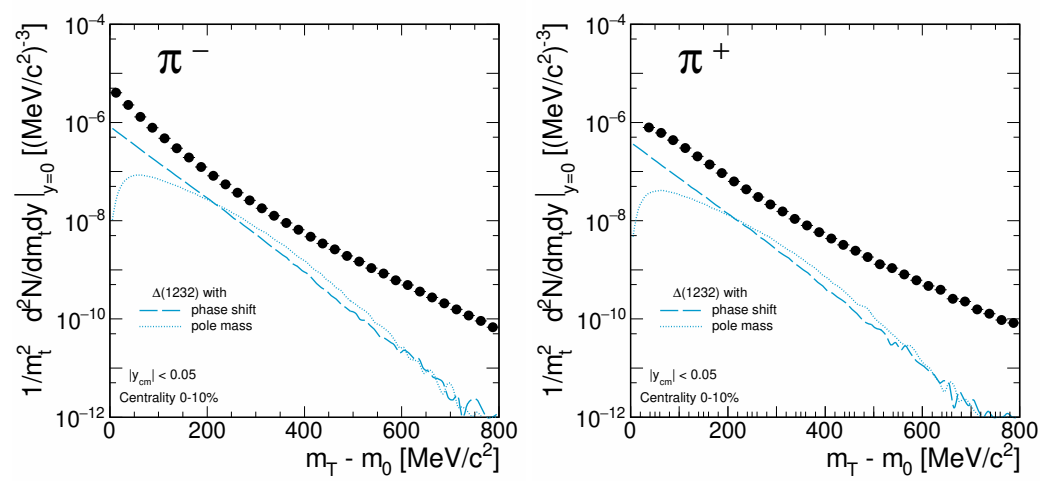

Figure 1. Transverse mass distributions of $\pi^{-}$(left panel) and $\pi^{+}$(right panel) at mid-rapidity, measured by HADES [4] overlaid with contribution from decays of $\Delta(1232)$, calculated with mass distribution from [10] (blue solid curves) and with zero width at nominal mass (blue dashed curves).

of $\pi^{ \pm}$just to the contribution from decays of $\Delta(1232)$, either having correct mass distribution (blue dashed curves) or zero width (blue solid curves), which was the only option available in THERMINATOR by now. The difference in shape is clear. However, with relatively low temperature that we obtained as discussed above, the yield of $\Delta$ is low and the difference does not influence the final results. The situation would be different at slightly higher temperature, where $\Delta$ would not be suppressed so strongly by the thermal factor. 


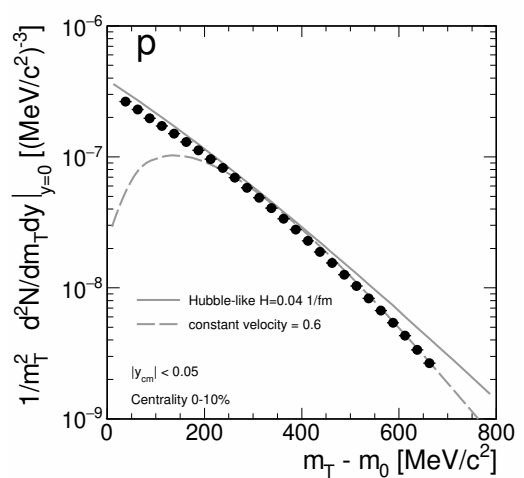

Figure 2. Mid-rapidity transverse mass distribution of protons, measured by HADES [5], compared to thermal model calculations with parameters from [11] and best fits of the fireball radial expansion velocity, assuming either Hubble-like profile $\beta(r)=\tanh (H r)$ (blue solid curve) or constant velocity (blue dashed curve).

The role of the shape of radial expansion velocity profile is illustrated in Fig. 2. The solid line shows the best fit with Hubble-like expansion, the dashed line - with constant velocity, independent of the radial position. In the latter case the model curve tends to be more concave and has a chance to meet data points only at high $m_{t}$ at the cost of missing them badly at low $m_{t}$. Such a fit gives also rise to an unexpectedly high expansion velocity of $\beta \sim 0.6$.
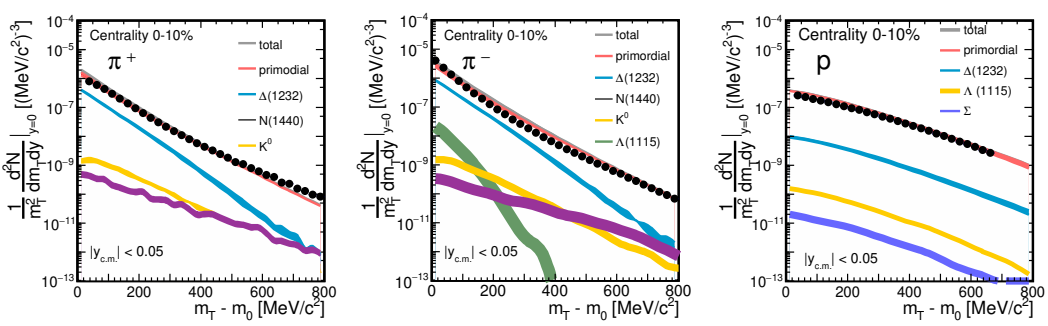

$\mathrm{T}=49.6 \mathrm{MeV}$

$\mu_{\mathrm{B}}=776 \mathrm{MeV}$

$\mu_{\mathrm{L}_{\mathrm{s}}}=-14.1 \mathrm{MeV}$

$\mu_{\mathrm{S}}=123.4 \mathrm{MeV}$

$\gamma_{\mathrm{s}}=0.16$

$\mathrm{R}=16.02 \mathrm{fm}$

$\mathrm{H}=0.041 / \mathrm{fm}$
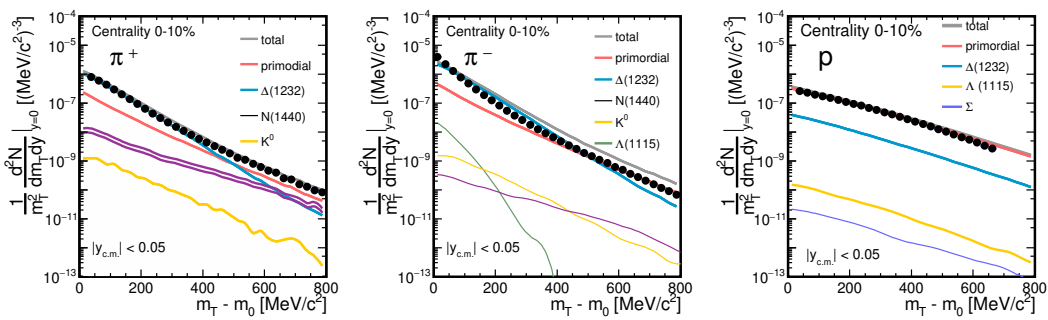

$\mathrm{T}=70.3 \mathrm{MeV}$

$\mu_{\mathrm{B}}=876 \mathrm{MeV}$

$\mu_{\mathrm{b}}=-21.5 \mathrm{MeV}$

$\mu_{\mathrm{S}}=198.3 \mathrm{MeV}$

$\gamma_{\mathrm{s}}=0.05$

$\mathrm{R}=6.01 \mathrm{fm}$

$\mathrm{H}=0.0971 / \mathrm{fm}$

Figure 3. Transverse mass distributions of $p$ and $\pi^{ \pm}$at mid-rapidity, measured by HADES, compared to thermal model calculations with parameters from [11] (upper row) and from [12], corresponding to the same way of treating nuclear clusters, but to the higher-temperature minimum of the thermal fit (lower row, for details see text and the reference). The respective values of the parameters are also shown.

Particle multiplicities measured by HADES have also been studied recently in ref. [12]. Several assumptions about the cluster formation have been tested there and in the setup equivalent to ours (implicit assumption of the nuclear coalescence) two degenerate minima of the thermal fit have been found. The first one results in thermal parameters consistent with the ones in [11], while the other one gives substantially higher freeze-out temperature and density. In the present work, we used THERMINATOR to generate particle spectra for the latter set of thermal parameters and the corresponding expansion coefficient $H$, which we extract in the same way as before. The resulting mid-rapidity $m_{t}$ spectra of protons and pions are com- 
pared to the experimental data in the lower part of Fig. 3 and contrasted with the case of ref. [11] shown in the upper part of the same figure. It can be observed, that a higher temperature leads to larger relative contribution of the $\Delta$ resonance and makes the proper treatment of its spectral shape indispensable. The overall agreement of the model with experimental data is good in both cases, except that for the higher temperature it clearly overshoots $\pi^{-}$yield at high $m_{t}$. At the same time, the slope of pion spectra above $m_{\mathrm{T}}-m_{0}=400 \mathrm{MeV} / c^{2}$ is perhaps better reproduced with higher $T$. By construction, the total particle multiplicities, given by integrals of their distributions over the full phase space, agree exactly with the experiment for both sets of parameters. In all cases, particle abundances provide not sufficient information to verify the assumptions of the thermal model, especially at lower collision energies, where not many degrees of freedom are available. More comprehensive information is provided by the kinematic distributions of the particles, but in order to exclude one or another hypothesis, finer details of the model have to be considered.

\section{Conclusions and outlook}

Statistical hadronization together with proper treatment of the fireball expansion and resonance dynamics describes reasonably well particle spectra measured in heavy-ion collisions in the few-GeV energy regime. However there is room for further improvements. Spherical symmetry is not exact and more realistic parametrization of the fireball expansion has to be implemented. The same holds for actual inclusion of light nuclear clusters. The measured spectra of $\pi^{+}$and $\pi^{-}$at low $m_{t}$ are clearly modified by a final-state electromagnetic interaction. An event generator allows for introduction of quantum-mechanical correlations and study of various effects observed in femtoscopic measurements. A model developed in this way can be compared with recent data from STAR fixed-target program and from future facilities, like FAIR and NICA.

\section{Acknowledgements}

This work was supported in part by: the Polish National Science Center Grants No. 2016/23/B/ST2/00717, No. 2018/30/E/ST2/00432, and No. 2017/26/M/ST2/00600; TU Darmstadt, Darmstadt (Germany), Helmholtz VH-NG-823, DAAD PPP Polen 2018/57393092; Goethe-University, Frankfurt(Germany), and HIC for FAIR (LOEWE).

\section{References}

[1] A. Andronic and others, Nature 561, 321 (2018)

[2] M. Chojnacki and others, Comput. Phys. Comm. 103, 746-773 (2012)

[3] W. Broniowski, W. Florkowski, Phys. Rev. Lett. 87, 272302 (2001)

[4] HADES Collaboration, Eur. Phys. J. A 56, 250 (2020)

[5] M. Szala (HADES Collaboration), in Proceedings of the XVIII International Conference on Strangeness in Quark Matter (SQM 2019)

[6] F. Cooper, G. Frye, Phys. Rev. D 10, 186 (1974)

[7] HADES Collaboration, Phys. Lett. B 793, 457 (2019)

[8] HADES Collaboration, Phys. Lett. B 778, 403 (2018)

[9] P. J. Siemens, J. O. Rasmussen, Phys. Rev. Lett. 42, 880 (1979)

[10] P. M. Lo and others, Phys. Rev. C 96, 015207 (2017)

[11] S. Harabasz and others, Phys. Rev. C 102, 054903 (2020)

[12] A. Motornenko and others, arXiv:2104.06036 [hep-ph] (2021) 